\title{
Modern Pulsometer for Traditional Indian Medicine
}

\author{
B.Persis Urbana Ivy \\ Assistant Professor, SITE, \\ VIT University, Vellore, \\ India 632014. \\ Research Scholar, \\ Sathyabama University, \\ Chennai, India.
}

\author{
Dr.R.Rani Hema Malini \\ Prof \& Head,E\&I Dept, \\ St.Peter's University, \\ Chennai, India 600054.
}

\begin{abstract}
Siddha is a natural treatment and the oldest medical system of healing in the world. Nadi which is a pulsebased diagnosis method which is the skill of feeling the pulse, and is known to dictate all the salient features of a human body. In this paper, we provide a complete spectrum of details of our procedure for obtaining three different pulses based on time series. This system contains contains a strain gauge equipped with a diaphragm element, a transmitter and also an amplifier, a digitizer which quantifies the analog signals. The system is designed with 16-bit accuracy in such a way that it provides no interference noise and no external electronics. Compared with the prior systems like ECG, the system provides a detailed classification of the nadi pulses which produces the waveforms with respect to abnormalities.

The varying pressure given to the pulse analyzer classifies vadha, pitha, and kapha based on the abnormalities captured from a single artery. The obtained output from this module is been fed to the knowledge management system that identifies the diseases based on body type. The designed system is being evaluated by siddha practitioners as a computeraided diagnostic tool
\end{abstract}

\section{General Terms}

Diaphragm, Digitizer, Pulse analyzer,

\section{Keywords}

Siddha, pulse analyzer, varying pressure.

\section{INTRODUCTION}

The Siddha system of medicine is the oldest in the world. It is one of the two ancient medicines in India. Siddha comes from the word Siddhi, means an object to attain perfection or heaven. Its known that the function of human body is being governed by the three most energetic forces: Vata, Pitta, and Kapha, These are three in number and are called as Tridosha. As shown in the Fig (I), the three different pulses are obtained. The Siddha medicine is cornerstone concept of the biological humors or Doshas.

They are the basic operating principles which govern the psycho-biological aspect of the body. These doshas are composed by the combination of the five elements or the 'PanchMahabhut'. The standard position to obtain Tridosha is through a "pulse waveform" obtained on a wrist with the index, middle and the ring fingers respectively.
These pulses which are obtained from the nadi are similar to the pulses from the ECG. According to the Indian traditional medical therapies, tridosha is the disproportion of three constituents in the body. These three constituents regulate the body functions and the imbalance leads to many disorders in the body system. Vatha, Pitha and kapha, are made up of five elements namely Earth, Water, Fire, Air and Space.

These co-exist in every cell of the human body and thus, function accordingly.

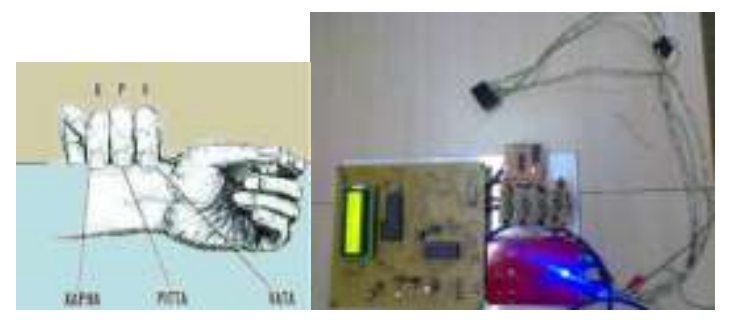

Fig ( I ): Obtaining a standard pulse in Siddha system of medicine.

- Vatham, made up of Space and Vayu, controls the nervous system.

- $\quad$ Pitham, formed by Fire, directs the metabolic activities of the body, digestion, absorption, heat, etc.

- Kapham, formed by Earth and Water, controls the permanence of the human body.

The imbalance of these doshas engraves various diseases in the body. In the Indian traditional medical therapies, the balance of the three doshas (humors) is compulsory to maintain a healthy state of min and body.The pulse analyzer captures the imbalances of tridosha in a non invasive method. Since it is inexpensive, convenient, and painless this system is considered efficient than the prior ones.This system captures the arterial pulse as a time series of the data. It has been checked with the properties of collected pulse waveforms based on the pressure applied on to obtain the pulses irrespective of the age.

The rest of the paper is as follows. After getting a general idea of the previously developed methods in Section II, we describe our system in Section III.

We compare our waveforms with previous methodologies, describe variations with respect to applied pressure and age in section IV and then we conclude with some final remarks. 


\section{PRIOR WORK}

Many procedures are available for measuring the electrical activity of the abnormalities of the body. some of them are the biomedical signal processing area, especially in the area of cardiology indicates that most of the disorders in heart can be completely captured in an Electrocardiogram(ECG). The elecroneurogram (ENG) is an electrical signal observed as a stimulus and the associated nerve action potential propagate over the length of a nerve, It may be used to measure the velocity of propagation (or conduction velocity) of a stimulus or action potential in a nerve. The electroencephalogram (EEG : popularly known as brain waves) represents the electrical activity of the brain. The phonocardiogram (PCG) is a vibration or sound signal related to the contractile activity of the cardiohemic system (the heart and blood together).The carotid signal (CP) is a pressure signal recorded over the carotid artery as it passes near the surface of the body at the neck. It provides a pulse signal indicating the variations in arterial blood pressure and volume.And many more methods are available for obtaining the abnormalities in the body.

There are many disadvantages associated with all these processes which can be overcome with the usage of the pulse analyzer which is proposed in this paper. The pulse that is obtained from the analyzer provides the reading of the Vatha, Pitha and kapha which are used to extract the similar disorders with in the body. Similar methodology, as already used in electro cardiology area, can be applied to the pulse waveforms to give a complete computer-aided system.

\section{OUR SYSTEM}

In this section, we describe the salient features of traditional Indian medicine. The pulses are sensed by the pressure exerted by the artery. The nadi pulses are sensed by the fingertip. The pulses obtained are very minute to analyze and very challenging too.

For this, the experiments were carried out using three pressure sensors, 'Millivolt Output Medium Pressure Sensor' [Mouser Electronics, Inc.] with tiny diaphragm at the center, which has the pressure range of ' $0-4$ inch $\mathrm{H} 2 \mathrm{O}$ '.

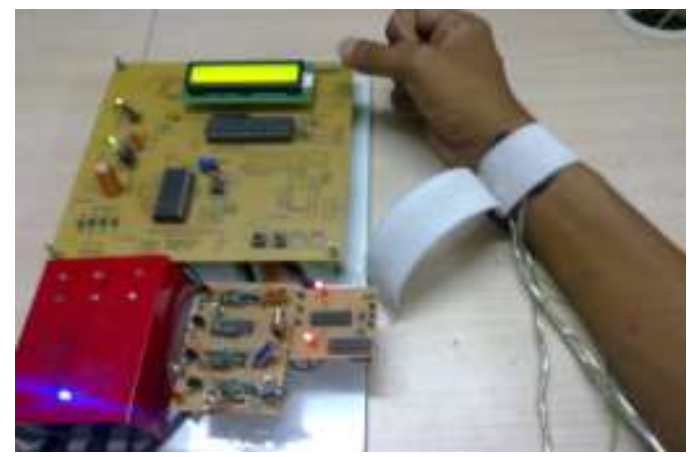

Fig ( II) : Pulse obtained from in our setup.

As shown in the above Fig,, The three location pulses namely vata, pitha, kapha are sensed by the set of three pressure transducers which is mounted on the wrist. The digitized output from the pressure sensing element is experienced by using the 16-bit multifunction data acquisition card NI USB-6210 (National Instruments, TX, USA). It has been interfaced with the personal Computer .The electrical signal is proportional to the pressure experienced, in differential modes.

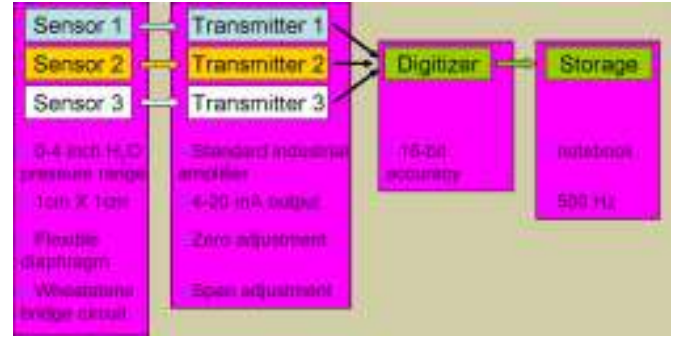

Fig (III). Hardware setup of our system.

A sampling rate of $500 \mathrm{~Hz}$ (higher than the Nyquist criteria) is been captured for a length of time which is predetermined. The strain gauge transducer is approximately of Dimension $1 \mathrm{~cm} \times 1 \mathrm{~cm}$. A flexible diaphragm is placed at the center, which is a forcegathering element.

The deformation is being done by arterial pressure waves. Three constant resistors are present in a Wheatstone bridge circuit along with the variable resistor transforms the strain output into an electrical signal which is proportional to it.A micro machine is been utilized by the sensor gives a stress concentration to the enhanced structure that provides linear output to the pressure measured.

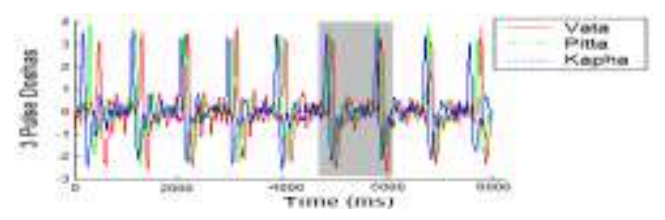

(a) Vata, pitta and kapha of a patient. Zoomed Gray portion indicates pitta dosha.

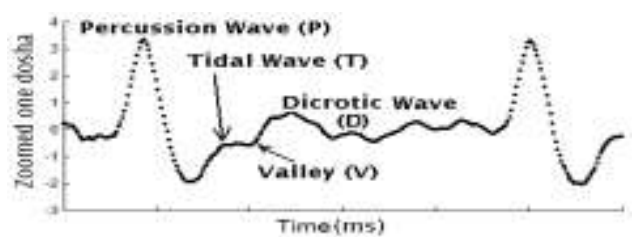

(b) Time-domain features in a pulse. The dotted style gives an idea of the number of points per pulse cycle.

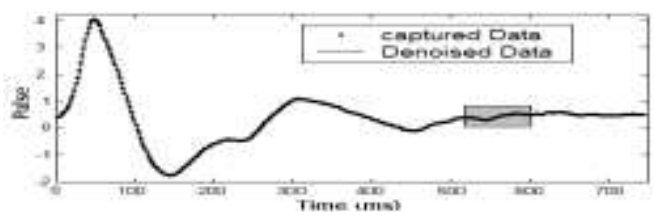

\section{( C )Denoising Using Wavelets}

Fig (III): Time domain features

To provide conditioned and linearised signal a standard transmitter and an amplifier with $4-20 \mathrm{~mA}$ is used. The 4$20 \mathrm{~mA}$ is converted to $2-10 \mathrm{~V}$ through a resistor of 500 ohms while connecting to 16-bit digitizer. The span and zero adjustments are calibrated with zero atmospheric 
pressure is provided to adjust the zero. The output from the zero pressure is $4 \mathrm{~mA}$. The output signal is delivered as an amplified signal which has been adjusted by the span. Due to explicit and implicit electrical and electronic noises, the data obtained are corrupted. In our system the noise level is negligible because of proper shielding.

\section{DISCUSSION}

Our system analyzes the waveforms obtained. Sample waveforms are obtained from the left hand artery of a patient. Section III shows the wave forms obtained which has been given in Fig.3. The respective doshas vata, pitha and kapha are obtained from three different pressure transducers . this has been represented in three different colours. The sampling frequency is $500 \mathrm{~Hz}$. The zoomed waveform is shown in Fig 3(b). It gives us the number of points per pulse cycle. It has been represented in the form of secondary peaks.

The extreme high component is used to remov the noise by wavelet denoising . sothat the obtained pulse is clean .(Fig 3(c)) shows the component . the denoised pulse, the solid line followed by the dotted line. This line indicates the pulse captured from sensors.

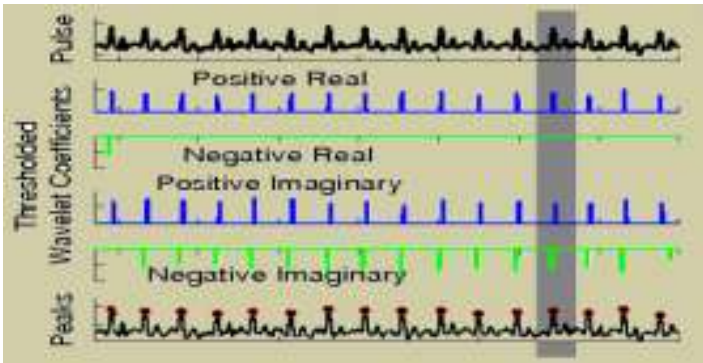

Fig (IV): Beat-to- Beat alteration

As shown in the Fig.3(b), The Time domain features are Percussion wave $(\mathrm{P})$, valley $(\mathrm{V})$, tidal wave $(\mathrm{T})$ and dicrotic wave (D). These three important time domain features are obtained from the pulse waveform. A standard pulse wave form with definite amplitude and time duration must be their in the wave-parts. This indicates that the body organs and the heart is functioned proper or not. The prior systems were compared with the collected waveform which is rich in harmonics and it is superior too.

\section{COMPARISON \\ WITH \\ EARLIER SYSTEMS.}

Few of the pulse waveforms obtained from earlier works is shown in Fig. 4. The least works has been carried out on different patients, of different age groups. In different resolutions and by using different techniques it has been mentioned in subfigures from (a) through(o) are from [4], [12], [13], [5], [14], [15], [16], [17], [18], [19], [20], [21],[22], [9], [23] respectively.

Observations were done with the previous improvements which is not a substantial improvement from 1950s till current methodologies. This method permits us to diagnosis the isorders from the wave forms obtained from the patients wrist. Machine learning algorithm is used for further extraction. This algorithm is used to classify the pulses depend not only on prior works but also on clinician's experience.

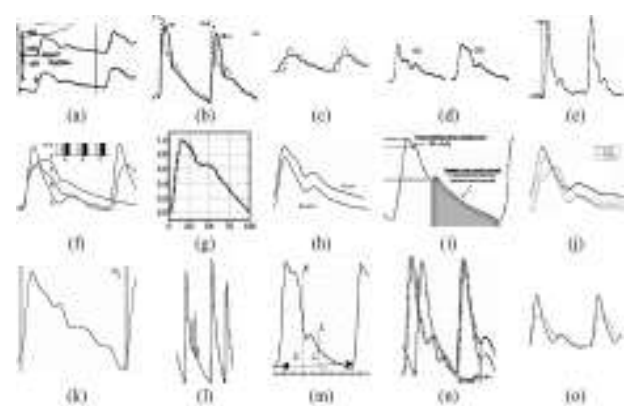

Fig ( V) This arrangement is based on ascending published dates and these Pulses are presented in selected earlier works.The knowledge management system will have the database of about the different disorders, symptoms based on the body type and the remedial actions to be taken which includes the food items should be followed. Here we have provided a distinct pattern for vata dosha observed from various patients.

As shown in Fig (VI): five different pulses obtained from different disorders. For example, The regular behavior of main and secondary peaks indicates a healthy pulse.In nature the fever pulse is not very often. Further, other disorders can be seen in the pulse wa e forms as we can see in the pictures. The amplitudes, the rising \& falling slopes, velocities, systolic \& diastolic energies and so on. There are major types of nadis which are prevailing in Siddha and Machine learning algorithms can be used on these waveforms. We can find some correlation between successive heart beats and it has a strong temporal similarities.

It is more appropriate in variability in pulses. The variation between consecutive beats is more important than the heart / pulse rate or the average values. Variability in various time-domain, frequency Domain morphology-based features;such as velocities, energies, entropies and amplitude have been explored [24], [9].This system captures the pulses in Such variability patterns.

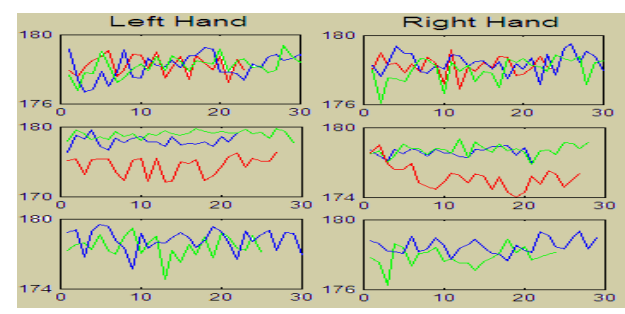

Fig. (VI). Cross-correlations are displayed between two doshas (vata and pitta) of left hand, and one dosha (vata) of both the hands of a patient.

The phase differences between the harmonics of Fast Fourier Transform can be determined as the phase difference. The reveals the cross-correlation features of our body conditions.

\section{IMPORTANT PROPERTIES OF PULSE WAVEFORM.}

Since or system gives accurate, reproduciable signal, the complex signals should also be provided in an 
accurate manner. The completeness and the reproduciablity of our system has been checked in the following manner:

A study has been conducted by recording the single healthy person $\mathrm{s}$ pulse wave form at different times from 4.00a.m to 5.00 p.m for ten subsequent days with the same settings. The Approximate Entropy (ApEn) of the waveforms was observed and the pulse cycles were checked and it was constant for the same timings for the ten days. Slight changes has been observed due to changes in stress ,thoughts and in mental status and so on.

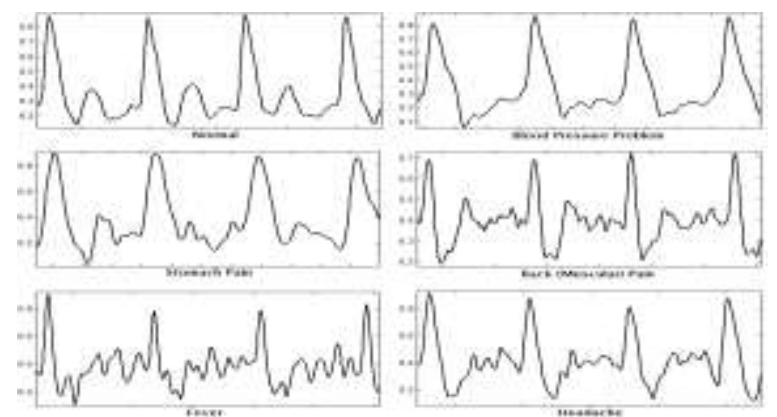

. Fig.(VI). various disorders have been captured on patients wrist.

The digitizer having an accuracy of 8-bit, 12bit and 16-bits. The acquired series has been checked for completeness. The pulse acquired with the same sensor was accurate. The capture details are accurate in 12- bit compared with 8 bit. The 16-bit details are more accurate.

\section{KNOWLEDGE MANAGEMENT SYSTEM}

In the siddha system of medicines the nadi is sensed at the wrist of the patient with varying pressure in different amplitudes and energies etc. This was correlated with the body conditions. The pulse vata, pitta and kapha sensed by the sensors are sent to the system for further remedies. The knowledge managemenet system has the classification of diseases according to the body type.

The vata , pita and kapha type diseases are stored in the databases. The vaiability in the pulse indicates that the dominant pulse is analysed and the rectification for which the symptoms are matched. These matching is done with the knowledge management system. The symptoms has been identified by the knowledge management system which in turn gives the rectification and the food items to be followed by the patient are displayed. This can further be send to the centralized system where the clinicians can verify and the priscribtions are generated.Variations in pressures and the mapping to KMS

As per our previous observations done the changes in the pulse waveforms for 31 patients in our database based on their age. They are classified in age groups 'below 25', '25-50' and 'above 50'. We have observed that the 'below 25 ' pulses are more dominant in secondary peaks. ' $25-50$ ' group is relatively stable, while older pulses are not regular in nature. We have given samples of healthy nadi of 3 groups in Fig. 8 of both the hands. As can be seen, the pulse duration goes up (rate decreases) as the age increases and also the patterns are different. Again, these observations are consistent with [4], [15]; the changes are indicative of an age-dependent reduction in large artery (capacitive) compliance and in small artery (oscillatory or reflective) compliance.

However, we consider all above findings to be only a preliminary suggestion to investigate further with much larger dataset. While applying machine learning algorithms on pulse dataset for identifying pulse type, we need to consider all the above dimensions, i.e. age, pressure applied, disorder and many more given in the literature.

Our system observes the pulses in all age groups, finds the variations and locates the correct disorders based on the symptoms observed. This can be accurate compared with the prior work done. Disorders may differ from person to person which can be captured through the our analyzer and the disease idenfication is done by our knowledge management system.

\section{CONCLUSION}

The Modern sensors reflect the feeling information which is being presently used in Indian ways of Siddha or the regular Traditional Chinese Medicine (TCM). We have taken the pressure sensing based methodology and the current progress in the instrumentation technologies in the desing of hight quality pulse acquisition system. Our Pulse waveforms, in the form of time series, has high details, as compared to the former reported systems.

We have proved our waveforms are reproducible and complete. We also showed variations with respect to applied age and pressure groups which are consistent with Siddha literature and prior work. Based on this, we believe that our system can be used by a larger number of lay persons. We are currently evaluating, with the help of Siddha practitioners the use of Nadi Tarangini for diagnostic purposes. Strong machine learning algorithms can be tried on these waveforms to differentiate them into major types of nadis defined in Siddha literature.

\section{REFERENCES}

[1] Aniruddha Joshi, Anand Kulkarni, Sharat Chandran, V. K. Jayaraman and B. D. Kulkarni: Nadi Tarangini: A Pulse Based Diagnostic System, pune, 2007.

[2] V. Lad, Secrets of the pulse: The ancient art of Ayurvedic pulse diagnosis. Motilal Banarsidass, Delhi, 2005.

[3] S. Upadhyay, Nadi vijnana (Ancient pulse science). Chaukhambha Sanskrit Pratishthan, Delhi, 1986.

[4] H. Warner, H. Swan, D. Connolly, R. Tompkins, and E. Wood, "Quantitation of beat-to-beat changes in stroke volume from the aortic pulse contour in man," Journal of Applied Physiology, vol. 5, pp. 495- 507, 1953.

[5] G. McVeigh, C. Bratteli, D. Morgan, C. Alinder, S. Glasser, S. Finkelstein, and J. Cohn, "Age-related abnormalities in arterial compliance identified by pressure pulse contour analysis: aging and arterial compliance," Hypertension, vol. 33, pp. 1392-1398, 1999.

[6] O. Lau and A. Chwang, "Relationship between wristpulse characteristics and body conditions," In Proc. of the 14th Engineering Mechanics Conf., 2000. 
[7] A. Brumfield and M. Andrew, "Digital pulse contour analysis: investigating age-dependent indices of arterial compliance," Physiological Measurement, vol. 26, pp. 599-608, 2005.[8] L. Xu, D. Zhang, K. Wang, N. Li, and $\mathrm{X}$. Wang, "Baseline wander correction in pulse waveforms using wavelet-based cascaded adaptive filter," Computers in Biology and Medicine, vol. 37, pp. 716-731, 2007.

[9] L. Xu, K. Wang, L. Wang, and N. Li, "Pulse contour variability before and after exercise," IEEE CBMS, 2006.

[10] M. Aritomo and Y. Yonezawa, "A wrist-mounted activity and pulse recording system," In Proc. of the First Joint BMES/EMBS Conf., vol. 2, p. 693, 1999.

[11] H. Lee, S. Suzuki, Y. Adachi, and M. Umeno, "Fuzzy theory in traditional chinese pulse diagnosis," In Proc. of Int. Joint Conf. on

Neural Networks, vol. 1, pp. 774-777, 1993.

[12] J. Remington and E. Wood, "Formation of peripheral pulse contour in man," Journal of Applied Physiology, vol. 9, pp. 433-442, 1956.

[13] J. S. K.H. Wesseling, J.R. Jansen and J. Schreuder, "Computation of aortic flow from pressure in humans using a nonlinear, three-element model," Journal of Applied Physiology, vol. 74, pp. 2566-2573, 1993.

[14] Y. Yoon, M. Lee, and K. Soh., "Pulse type classification by varying contact pressure," IEEE Engineering in Medicine and Biology Magazine, vol. 19, pp. 106-110., 2000.

[15] N. Linton and R. Linton, "Estimation of changes in cardiac output from the arterial blood pressure waveform in the upper limb," British Journal of Anaesthesia, vol. 86, pp. 486-496, 2001.
[16] J. Allen and A. Murray, "Age-related changes in the characteristics of the photoplethysmographic pulse shape at various body sites." Physiological measurement, vol. 24, pp. 297-307, 2003.

[17] C. Vlachopoulos, N. Alexopoulos, D. Panagiotakos, M. O'Rourke, and C. Stefanadis, "Cigar smoking has an acute detrimental effect on arterial stiffness," American Journal of Hypertension, vol. 17, pp. 299-303, 2004.

[18] D. Duprez, D. Kaiser, W. Whitwam, S. Finkelstein, A. Belalcazar, R. Patterson, S. Glasser, and J. Cohn, "Determinants of radial artery pulse wave analysis in asymptomatic individuals,"American Journal of Hypertension, vol. 17, pp. 647-653, 2004.

[19] L. Xu, K. Wang, and L. Wang, "Pulse waveforms classification based on wavelet network," IEEE EMBS, pp. 4596-4599, 2005.

[20] E. Treo, M. Herrera, and M. Valentinuzzi, "Algorithm for identifying and separating beats from arterial pulse records," BioMedical Engineering OnLine, vol. 4, 2005.

[21] L. Wang, K. Wang, and L. Xu, "Lempel-ziv decomposition based arrhythmic pulses recognition," IEEE EMBS, pp. 4606-4609, 2005.

[22] H. Wang and Y. Cheng, "A quantitative system for pulse diagnosis in traditional chinese medicine," IEEE EMBS, pp. 5676-5679, 2005.

[23] I. Wilkinson, C. McEniery, and J. Cockcroft, "Atenolol and cardiovascular risk: an issue close to the heart," The Lancet, vol. 367, pp. 627-629, 2006.

[24] L. Li and Z. Wang, "Study on interval variability of arterial pulse," In Proc. of the First Joint BMES/EMBS Conf., vol. 1, p. 223, 1999. 